\title{
Tau leptons from Earth skimming tau neutrinos
}

\author{
Jie-Jun Tseng* \\ Institute of Physics, Academia Sinica, Taipei 115, Taiwan \\ E-mail: 'gen@phys. sinica. edu.twi
}

\section{Tsung-Wen Yeh}

National Tai-Chung Teachers College, Taichung 403, Taiwan

E-mail: twwyeh@ms 3 ntctc. edu.twi

\section{H. Athar}

Physics Division, National Center for Theoretical Sciences, Hsinchu 300, Taiwan E-mail: 'athar@phys.cts.nthu.edu.tw'

\section{A. Huang}

National United University, Miaoli 360, Taiwan

E-mail: huangmh@phys ntu. edu.tww

\section{Fei-Fain Lee and Guey-Lin Lin}

Institute of Physics, National Chiao-Tung University, Hsinchu 300, Taiwan

E-mail: 'u8727515@cc.nctu.edu.tw, glin@cc.nctu.edu.twi

\begin{abstract}
A semi-analytic approach is developed for dealing with the tau-lepton flux emerging from the Earth, induced by the incident high energy tau neutrinos interacting inside the Earth for $10^{5} \leq E_{\nu} / \mathrm{GeV} \leq 10^{10}$. We obtain results for the energy dependence of the tau-lepton flux coming from the Earth-skimming neutrinos due to the neutrinonucleon charged-current interaction. We illustrate our results for several anticipated high energy astrophysical neutrino sources such as the AGNs, the GRBs, and the GZK neutrino fluxes. The tau lepton fluxes resulting from rock-skimming and ocean-skimming neutrinos are compared. Such comparisons might render useful information for the spectral indices of incident neutrino fluxes.
\end{abstract}

\section{Introduction}

To detect the high energy neutrinos $\left(E_{\nu}>10^{5} \mathrm{GeV}\right)$ is crucial to identify the extreme energy sources in the Universe, and possibly to unveil the puzzle of cosmic rays with

${ }^{*}$ Speaker. 
energy above the GZK cutoff[[i]1]. These proposed scientific aims are well beyond the scope of the conventional high energy gamma-ray astronomy. Because of the expected small flux of the high energy neutrinos, large scale detectors $\left(\geq 1 \mathrm{~km}^{2}\right)$ are required to obtain the first evidence.

The air showers caused by the charged leptons produced from the neutrino-nucleon scatterings taking place inside the Earth or in the air can be identified by the Cherenkov or the fluorescence detectors. It thus include the possibility of detection of quasi horizontal incident neutrinos which are also referred to as the Earth-skimming neutrinos. These neutrinos are considered to interact below the horizon of an Earth based surface detector.

To Measure the Earth-skimming neutrinos is proposed only recently [2]. The Pierre Auger observatory group has simulated the anticipated detection of the air-showers from the decays of $\tau$ leptons [i]. The tau air-shower event rates resulting from the Earth-skimming tau neutrinos for different high energy neutrino telescopes are given in

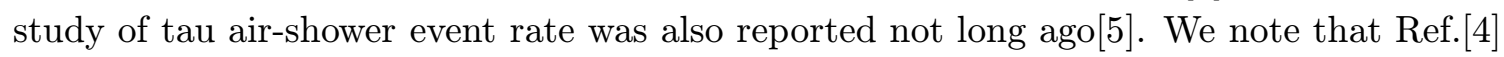
does not consider the tau-lepton energy distribution in the $\nu_{\tau}$-nucleon scattering, and only the incident tau neutrinos with energies greater than $10^{8} \mathrm{GeV}$ are considered. For Ref. [.5i], we note that only the sum of tau air-shower event rates arising from different directions is given. Hence some of the events may be due to tau-leptons/neutrinos traversing a large distance. As a result, it is not possible to identify the source of tau-neutrino flux even with the observation of tau-lepton induced air-shower.

In this work, we shall focus on the high energy Earth-skimming neutrinos and shall calculate the energy spectrum of their induced tau-leptons, taking into account the inelasticity of neutrino-nucleon scatterings and the tau-lepton energy loss in detail. Our work differs from Ref.[潭] by our emphasis on the Earth-skimming neutrinos. We shall present our results in the form of outgoing tau-lepton spectra for different distances inside the rock, instead of integrating the energy spectra. As will be demonstrated, such spectra are insensitive to the distances traversed by the Earth-skimming $\nu_{\tau}$ and $\tau$. They are essentially determined by the tau lepton range. Because of this characteristic feature, our results are useful for setting up simulations with specifically chosen air-shower content detection strategy, such as detection of the Cherenkov radiation or the air fluorescence. Our results are also beneficial for the coherent Cherenkov radio emission measurement detectors such as the Radio Ice Cherenkov Experiment (RICE) [후히 and the upcoming ANtarctic Impulsive

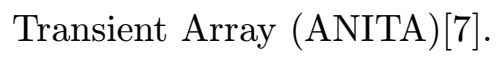

We start with our semi-analytic description in Section II and III. The transport equations governing the evolutions of neutrino and tau-lepton fluxes will be derived. In Section IV, we summarize our main results, namely the tau-lepton energy spectra due to neutrino-nucleon scatterings. The implications of our results will be discussed here also. In particular, we shall point out that the ratio of tau-lepton flux induced by rock-skimming neutrinos to that induced by ocean-skimming neutrinos is sensitive to the spectral index of the incident tau-neutrino flux. The summary will be given in Section V. 


\section{The tau lepton energy spectrum}

Considering only the neutrino-nucleon scatterings, the transport equations for tau neutrinos and tau leptons can be written as

$$
\frac{\partial F_{\nu_{\tau}}(E, X)}{\partial X}=-\frac{F_{\nu_{\tau}}(E, X)}{\lambda_{\nu_{\tau}}(E)}+n_{N} \sum_{i=1}^{3} \int_{y_{\min }^{i}}^{y_{\max }^{i}} \frac{\mathrm{d} y}{1-y} F_{i}\left(E_{y}, X\right) \frac{\mathrm{d} \sigma_{\nu}^{i}}{\mathrm{~d} y}\left(y, E_{y}\right),
$$

and

$$
\begin{aligned}
\frac{\partial F_{\tau}(E, X)}{\partial X} & =-\frac{F_{\tau}(E, X)}{\lambda_{\tau}^{\mathrm{cc}}(E)}-\frac{F_{\tau}(E, X)}{\rho d_{\tau}(E)}+\frac{\partial\left[(\alpha(E)+\beta(E) E) F_{\tau}(E, X)\right]}{\partial E} \\
& +n_{N} \int_{y_{\min }}^{y_{\max }} \frac{\mathrm{d} y}{1-y} F_{\nu_{\tau}}\left(E_{y}, X\right) \frac{\mathrm{d} \sigma_{\nu_{\tau} N \rightarrow \tau Y}}{\mathrm{~d} y}\left(y, E_{y}\right),
\end{aligned}
$$

where $n_{N}$ the number of target nucleons per unit medium mass, and $\rho$ is the mass density of the medium. The $\sigma_{\nu}^{1,2,3}$ are defined as $\sigma\left(\nu_{\tau}+N \rightarrow \nu_{\tau}+Y\right), \Gamma\left(\tau \rightarrow \nu_{\tau}+Y\right) / c \rho n_{N}$, and $\sigma\left(\tau+N \rightarrow \nu_{\tau}+Y\right)$ respectively. The quantity $X$ represents the slant depth traversed by the particles, i.e., the amount of medium per unit area traversed by the particle (and thus in units of $\mathrm{g} / \mathrm{cm}^{2}$ ). The $\lambda_{\nu}, d_{\tau}$, and $\lambda_{\tau}^{\mathrm{CC}}$ represent the $\nu_{\tau}$ interaction thickness, the taulepton decay length, and the tau-lepton charged-current interaction thickness respectively, with, say, $\lambda_{\nu}=1 /\left(n_{N} \sigma_{\nu N}\right)$ and $d_{\tau}=c \tau_{\tau} E / m_{\tau}$. The $E_{y}$ is equal to $E /(1-y)$, where $y$ is the inelasticity of neutrino-nucleon scatterings, such that the initial and final-state particle energies in the differential cross sections $\mathrm{d} \sigma_{\nu}^{i}\left(y, E_{y}\right) / \mathrm{d} y$ and $\mathrm{d} \sigma_{\nu_{\tau} N \rightarrow \tau Y}\left(y, E_{y}\right) / \mathrm{d} y$ are $E /(1-$ $y)$ and $E$ respectively. The limits for $y, y_{\min }^{i}$ and $y_{\max }^{i}$ depend on the kinematics of each process. Finally, the energy-loss coefficients $\alpha(E)$ and $\beta(E)$ are defined by $-\mathrm{d} E / \mathrm{d} X=$ $\alpha(E)+\beta(E) E$ with $E$ the energy being the tau lepton. An equation similar to Eq. (2.2. in the context of atmospheric muons was found in Ref. [8 8 in].

Before solving the above coupled transport equations, it is essential to know the energyloss coefficients $\alpha(E)$ and $\beta(E)$. As pointed out before $[\overline{9}]$, the coefficient $\alpha(E)$ is due to the

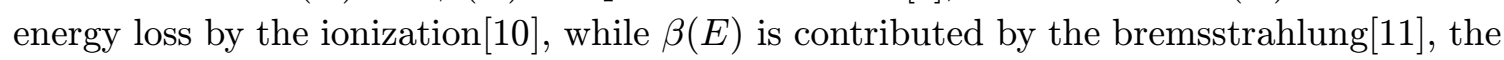

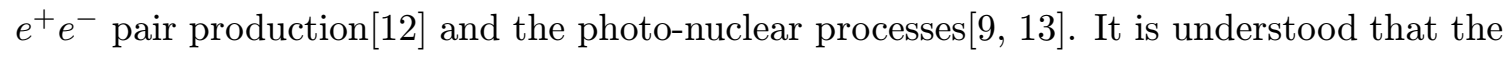
contribution by $\alpha(E)$ becomes unimportant for $E \geq 10^{5} \mathrm{GeV}$. The coefficient $\beta(E)$ can be parameterized as $\beta(E)=\left(1.6+6\left(E / 10^{9} \mathrm{GeV}\right)^{0.2}\right) \times 10^{-7} \mathrm{~g}^{-1} \mathrm{~cm}^{2}$ in the standard rock for $10^{5} \leq E / \mathrm{GeV} \leq 10^{12}$.

The tau-lepton flux can then be calculated from Eqs. $(\overline{2} \cdot \overline{1})$ and $\left(\overline{2} \cdot \overline{2} \overline{2}^{*}\right)$ once the incoming $\nu_{\tau}$ flux is given. The $\nu_{\tau}$ flux can be obtained by the following ansatz[i] $\left.{ }_{-1}^{\overline{4}}\right]$ :

$$
F_{\nu_{\tau}}(E, X)=F_{\nu_{\tau}}(E, 0) \exp \left(-\frac{X}{\Lambda_{\nu}(E, X)}\right),
$$

where $\Lambda_{\nu}(E, X) \equiv \lambda_{\nu}(E) /\left(1-Z_{\nu}(E, X)\right)$, with the factor $Z_{\nu}(E, X)$ arising from the regeneration effect of the $\nu_{\tau}$ flux. On the other hand, the tau-lepton flux is given by

$$
\begin{aligned}
F_{\tau}(E, X) & =\int_{0}^{X} \mathrm{~d} T G_{\nu}(\bar{E}, T) \\
& \times \exp \left[\int_{T}^{X} \mathrm{~d} T^{\prime}\left(\gamma^{\prime}(\bar{E})-\frac{1}{\rho d_{\tau}(\bar{E})}-\frac{1}{\lambda_{\tau}^{\mathrm{cc}}(\bar{E})}\right)\right],
\end{aligned}
$$


with $\bar{E} \equiv \bar{E}(X-T ; E)$, and

$$
G_{\nu}(E, X)=n_{N} \int_{y_{\min }}^{y_{\max }} \frac{\mathrm{d} y}{1-y} F_{\nu}\left(E_{y}, X\right) \frac{\mathrm{d} \sigma_{\nu_{\tau} N \rightarrow \tau Y}}{\mathrm{~d} y}\left(y, E_{y}\right) .
$$

It is easy to see that the factor $Z_{\nu}(E, X)$ enters into the expression for $F_{\tau}(E, X)$ through the function $G_{\nu}(E, X)$. Similarly, $Z_{\nu}(E, X)$ also depends on $F_{\tau}(E, X)$. It is possible to solve for $Z_{\nu}(E, X)$ and $F_{\tau}(E, X)$ simultaneously by the iteration method [i] $\left.{ }_{1}^{1} \overline{4}\right]_{1}$.

\section{Tau lepton range}

To obtain the tau-lepton range by our semi-analytic approach, we rewrite Eq. (12.2i) by dropping the neutrino term, i.e.,

$$
\frac{\partial F_{\tau}(E, X)}{\partial X}=-\frac{F_{\tau}(E, X)}{\lambda_{\tau}^{\mathrm{cc}}(E)}-\frac{F_{\tau}(E, X)}{\rho d_{\tau}(E)}+\frac{\partial\left[\gamma(E) F_{\tau}(E, X)\right]}{\partial E},
$$

with $\gamma(E) \equiv \alpha(E)+\beta(E) E$. One can easily solve it for $F_{\tau}(E, X)$ :

$$
F_{\tau}(E, X)=F_{\tau}(\bar{E}, 0) \exp \left[\int_{0}^{X} \mathrm{~d} T\left(\gamma^{\prime}(\bar{E})-\frac{1}{\rho d_{\tau}(\bar{E})}-\frac{1}{\lambda_{\tau}^{\mathrm{cc}}(\bar{E})}\right)\right],
$$

where $\bar{E} \equiv \bar{E}(X ; E)$ with $\mathrm{d} \bar{E} / \mathrm{d} X=\gamma(\bar{E})$ and $\bar{E}(0 ; E)=E$. To calculate the tau-lepton range, we substitute $F_{\tau}(E, 0)=\delta\left(E-E_{0}\right)$. The survival probability $P\left(E_{0}, X\right)$ for a taulepton with an initial energy $E_{0}$ at $X=0$ is

$$
P\left(E_{0}, X\right)=\frac{\gamma\left(\tilde{E}_{0}\right)}{\gamma\left(E_{0}\right)} \exp \left[\int_{0}^{X} \mathrm{~d} T\left(\gamma^{\prime}\left(\tilde{E}_{0}\right)-\frac{1}{\rho d_{\tau}\left(\tilde{E}_{0}\right)}-\frac{1}{\lambda_{\tau}^{\mathrm{cc}}\left(\tilde{E}_{0}\right)}\right)\right],
$$

where $\tilde{E}_{0} \equiv \tilde{E}\left(X ; E_{0}\right)$ with $\mathrm{d} \tilde{E}_{0} / \mathrm{d} X=-\gamma\left(\tilde{E}_{0}\right)$ and $\tilde{E}_{0}\left(0 ; E_{0}\right)=E_{0}$. The tau-lepton range is simply

$$
R_{\tau}\left(E_{0}\right)=\int_{0}^{\infty} \mathrm{d} X P\left(E_{0}, X\right) .
$$

For $E_{0}=10^{9} \mathrm{GeV}$, we find that $R_{\tau}=10.8 \mathrm{~km}$ in the standard rock $(Z=11, A=22)$ while $R_{\tau}=5.0 \mathrm{~km}$ in the iron. Both values are in good agreement with those obtained by Monte-Carlo calculations $\left[\bar{q}_{1}\right]$. To compare the tau-lepton ranges, we have followed the

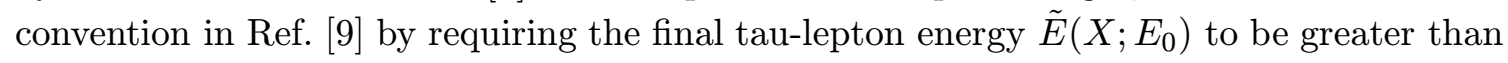
$50 \mathrm{GeV}$.

It is to be noted that we obtain $R_{\tau}$ by using the continuous tau-lepton energy-loss approach, rather than stochastic approach adopted in Ref. $[\overline{9} \overline{9}]$. In the muon case, the continuous approach to the muon energy loss is known to overestimate the muon range [1] Such an overestimate is not significant in the tau-lepton case, because of the decay term in Eq. (13.3. In fact, tau lepton decay term dictates the tau range in the rock until $E_{\tau} \geq 10^{7}$ $\mathrm{GeV}$. Even for $E_{\tau}>10^{7} \mathrm{GeV}$, the tau lepton range is still not entirely determined by the tau-lepton energy loss. Hence different treatments on the tau-lepton energy loss do not lead to large differences in the tau-lepton range, in contrast to the case for the muon range. 


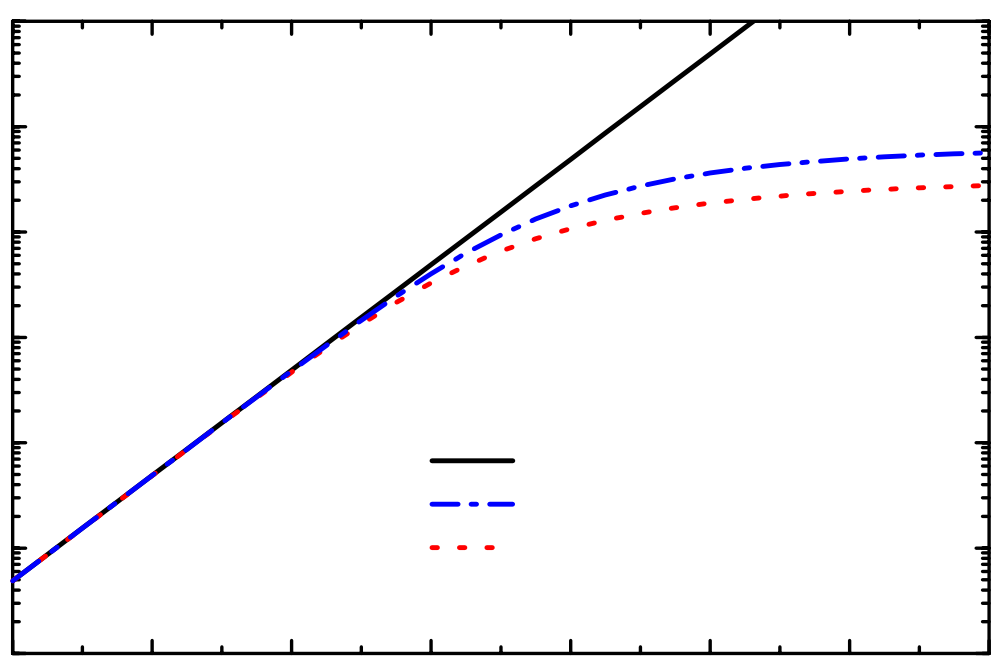

Figure 1: The tau-lepton range in rock and in water using Eq. [3. $d_{\tau}$ in $\mathrm{km}$ as a function of tau-lepton energy in $\mathrm{GeV}$.

Our result for the tau-lepton range up to $10^{12} \mathrm{GeV}$ are plotted in Fig. ${ }_{1}^{1}$. This is an extension of the result in Ref. [9]9], where the tau-lepton range is calculated only up to $10^{9}$ $\mathrm{GeV}$. Our extension is seen explicitly in the addition of charged-current scattering term on the R.H.S. of Eq. ( $\left(\overline{3} \cdot \overline{1}_{1}^{1}\right)$. This term is necessary because $1 / \lambda_{\tau}^{\mathrm{CC}}$ becomes comparable to $1 / \rho d_{\tau}$ in the rock for $E \geq 10^{10} \mathrm{GeV}$; whereas one does not need to include the contribution by the tau-lepton neutral-current scattering, since such a contribution can not compete

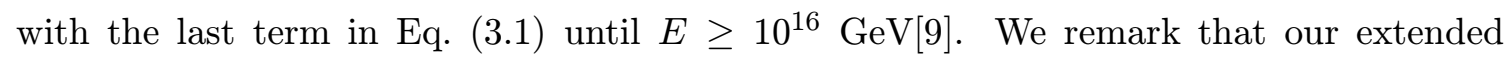
results for $R_{\tau}$ are subject to the uncertainties of neutrino-nucleon scattering cross section at high energies.

\section{Results and discussion}

In this section, we show the tau-lepton fluxes resulting from three kinds of diffuse astro-

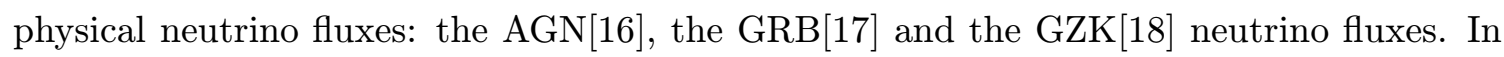
these representative models, $F_{\nu_{\tau}}$ arises because of neutrino flavor mixing [i] $\left.\overline{1}_{1}^{1}\right]$. Our convention for $F_{\nu_{\tau}}$ is that $F_{\nu_{\tau}}=\mathrm{d} N_{\nu_{\tau}} / \mathrm{d}\left(\log _{10} E\right)$ in the unit of $\mathrm{cm}^{-2} \mathrm{~s}^{-1} \mathrm{sr}^{-1}$. The same convention is used for the outgoing tau lepton fluxes. For completeness, let us remark here that the recent upper bound on diffuse astrophysical $F_{\nu_{\mu}}$ (not $F_{\nu_{\tau}}$ ) from Antarctic Muon And Neutrino Detector Array (AMANDA) B10 is of the order of $\sim 8.4 \cdot 10^{-7} \mathrm{~cm}^{-2} \mathrm{~s}^{-1}$ $\mathrm{sr}^{-1} \mathrm{GeV}$ for $6 \cdot 10^{3} \leq E_{\nu} / \mathrm{GeV} \leq 10^{6}[\overline{2} \overline{0} \overline{0}]$.

In Fig. '2i, and propagation of incident AGN and GRB neutrinos inside the rock $\left(\rho=2.65 \mathrm{~g} / \mathrm{cm}^{3}\right)$ for $X / \rho=10 \mathrm{~km}, 100 \mathrm{~km}$ and $500 \mathrm{~km}$ respectively. It is interesting to see that the tau-lepton energy spectra remain almost unchanged for the above three different slant depths/matter 


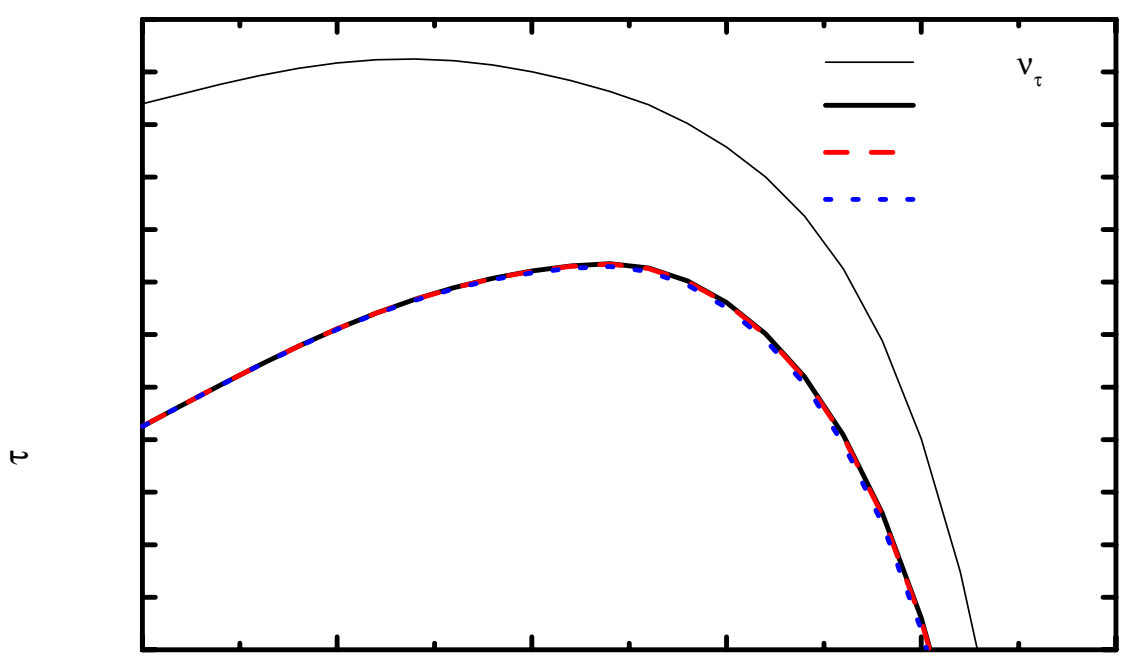

Figure 2: The tau-lepton energy spectrum induced by the AGN neutrinos in rock for three different $X / \rho$ ratio values. The incident tau-neutrino flux is shown by the thin solid line.

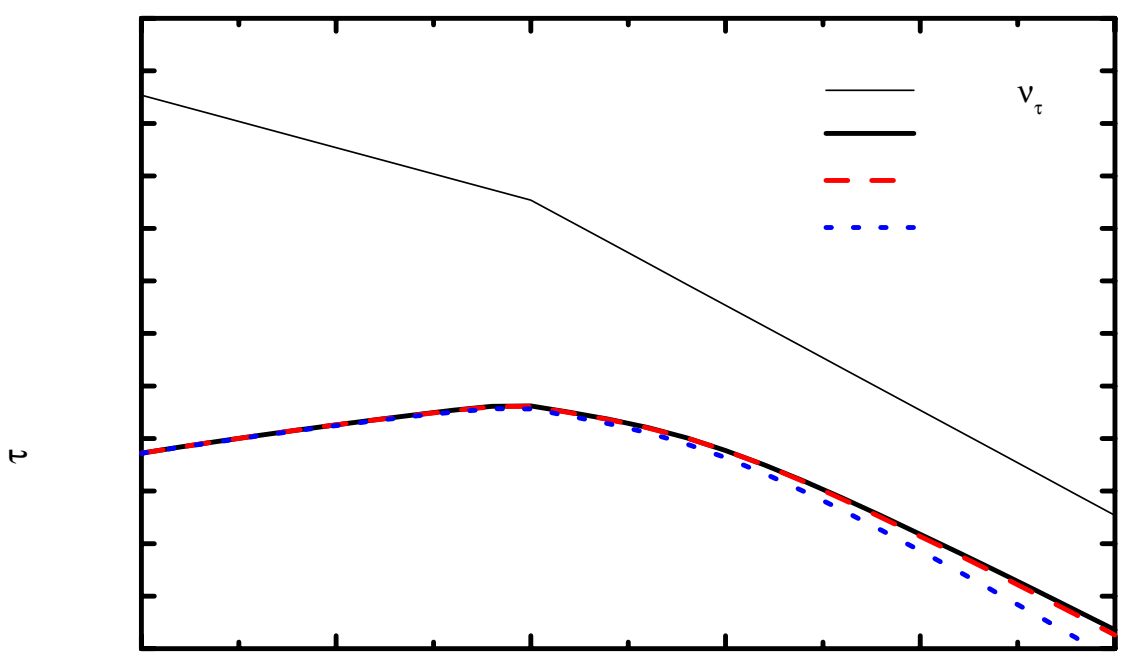

Figure 3: The tau-lepton energy spectrum induced by the GRB neutrinos in rock for three different $X / \rho$ ratio values. The incident tau-neutrino flux is shown by the thin solid line.

density ratio values. This feature can be understood by two simple facts. First of all, the neutrino-nucleon charged-current interaction length, which is related to the interaction thickness by $\lambda_{\mathrm{CC}}=\rho l_{\mathrm{CC}}$, is given by $l_{\mathrm{CC}}=2 \cdot 10^{4} \mathrm{~km}\left(\frac{1 \mathrm{~g} / \mathrm{cm}^{3}}{\rho}\right)\left(\frac{E_{\nu}}{10^{6} \mathrm{GeV}}\right)^{-0.363}$. Secondly, the tau leptons, which eventually exit the Earth, ought to be produced within a tau-leptonrange distance to the exit point. For a tau-lepton produced far away from the exit point, it loses energy and decays before reaching to the exit point. Hence the tau-lepton flux 
is primarily determined by the ratio of tau-lepton range to the charged current neutrinonucleon interaction length. The total slant depth $X$ which the tau-neutrino (tau-lepton) traverses inside the Earth is then unimportant, unless $X$ is large enough such that the tau neutrino flux attenuates significantly before tau-neutrino is converted into the tau-lepton.

On the other hand, a slight suppression is found for the GZK case at $E_{\tau}>10^{9} \mathrm{GeV}$ as one increases $X / \rho$ from $10 \mathrm{~km}$ to $500 \mathrm{~km}$ (see Fig. 燕). This is because the typical GZK tau neutrino flux peaks for energy range between $10^{7}$ and $10^{10} \mathrm{GeV}$, which corresponds to attenuation lengths ranging from $7,800 \mathrm{~km}$ down to $640 \mathrm{~km}$. One notices that $640 \mathrm{~km}$ is rather close to the distance $500 \mathrm{~km}$ which we choose for $X / \rho$. Hence a slight suppression on the tau-lepton flux occurs for $X / \rho=500 \mathrm{~km}$. We have compared our AGN-type tau-

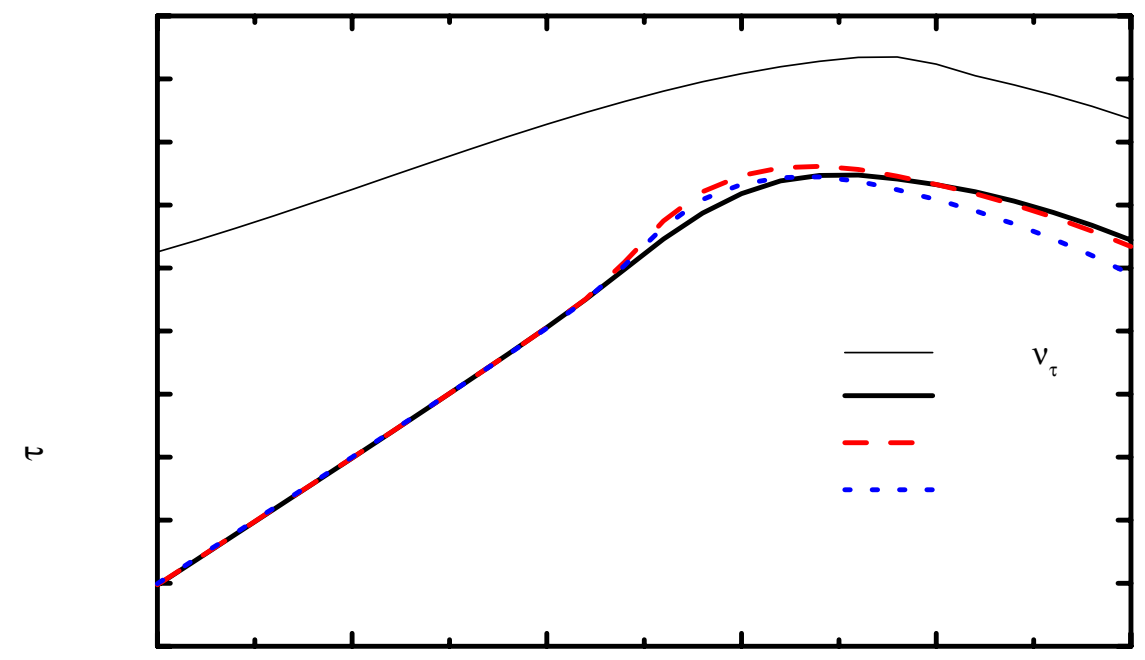

Figure 4: The tau-lepton energy spectrum induced by the GZK neutrinos in rock for three different $X / \rho$ ratio values. The incident tau-neutrino flux is shown by the thin solid line.

lepton flux with that obtained by Monte-Carlo simulations, adopting stochastic approach for the tau-lepton energy loss [in $2 \overline{1}]$. The two tau-lepton fluxes agree within $\sim 10 \%$. This is expected since the tau-lepton range obtained by the above two approaches agree well, as pointed out before.

It is possible that the tau-neutrino skims through a part of the ocean in addition to the Earth before exiting the interaction region[2를. Hence, it is desirable to compare the resulting tau-lepton fluxes as the tau neutrinos skim through mediums with different densities, while the slant depths of mediums are held fixed as an example. As stated before, the taulepton flux is essentially determined by the probability of $\nu_{\tau} N$ charged-current interaction happening within a tau-lepton range. Furthermore, from Fig. $11, i$ it is clear that the taulepton range equals to the tau-lepton decay length for $E_{\tau}$ less than $10^{7} \mathrm{GeV}$. One therefore expects $F_{\tau}^{\text {rock }}(E, X) / F_{\tau}^{\text {water }}(E, X)=\rho^{\text {rock }} / \rho^{\text {water }}$ for $E_{\tau}<10^{7} \mathrm{GeV}$. This is clearly seen to be the case from Fig. ${ }_{15}^{1}$, as we compare $F_{\tau}^{\text {rock }}$ with $F_{\tau}^{\text {water }}(E, X)$ for $X=2.65 \cdot 10^{6}$ $\mathrm{g} / \mathrm{cm}^{2}$. For $E_{\tau}>10^{7} \mathrm{GeV}$, the tau-lepton range has additional dependencies on the mass 


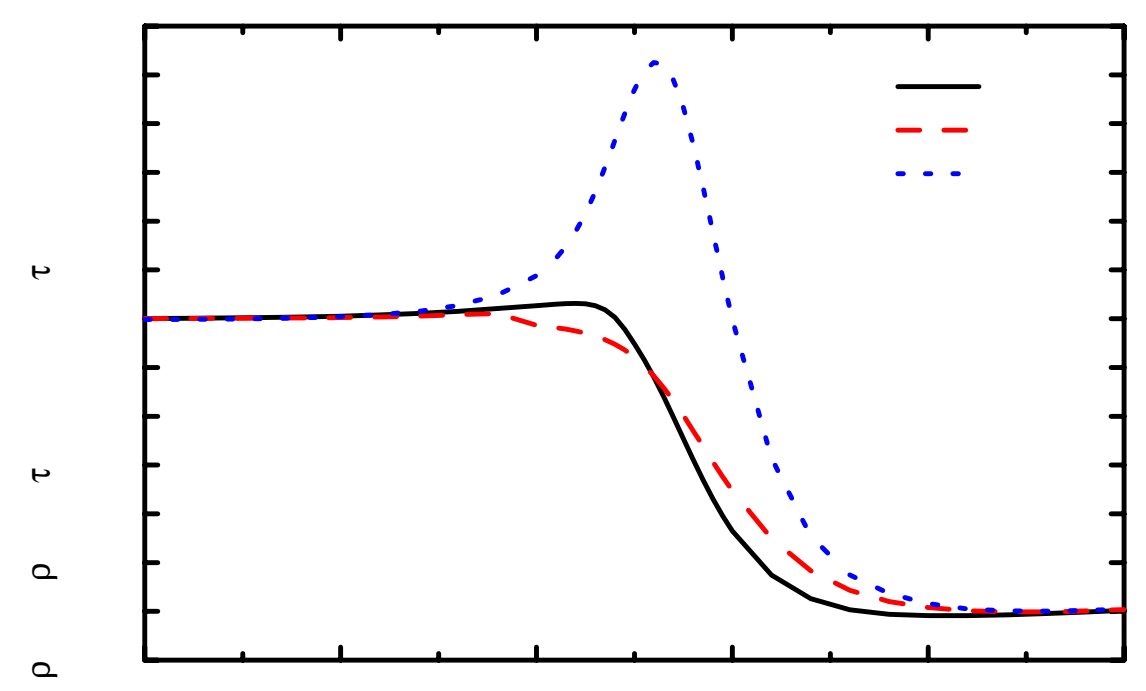

Figure 5: The ratio of $F_{\tau}$ in rock and water induced by the AGN, the GRB and the GZK neutrinos for $X=2.65 \cdot 10^{6} \mathrm{~g} / \mathrm{cm}^{2}$.

density and the atomic number of the medium. Hence the ratio $F_{\tau}^{\text {rock }}(E, X) / F_{\tau}^{\text {water }}(E, X)$ starts deviating from $\rho^{\text {rock }} / \rho^{\text {water }}$. It is worthwhile to mention that the tau-lepton flux ratios for AGN and GRB cases behave rather similarly. On the other hand, the ratio in the GZK case has a clear peak between $10^{7.5}<E / \mathrm{GeV}<10^{8.5}$. The appearance of this peak has to do with the relatively flat behavior of the incident GZK neutrino spectrum, while the position of this peak is related to the energy dependencies of the tau-lepton range and the neutrino-nucleon scattering cross sections. We have confirmed our observations by computing the flux ratios with simple power-law incident tau-neutrino fluxes. The above peak in the tau-lepton flux ratio implies the suppression of tau-lepton events from oceanskimming neutrinos compared to those from rock-skimming neutrinos. As stated earlier, the suppression of ocean-skimming neutrinos is related to the spectral index of the incident neutrino flux. It is therefore useful to perform a detailed simulation for it [2] a detailed study is needed because the slant depths traversed by the above two kinds of neutrinos are generally different.

\section{Summary}

In summary, we have given a semi-analytic treatment on the problem of simultaneous propagation of high energy tau neutrinos and tau leptons inside the Earth. Our treatment explicitly takes into account the inelasticity of neutrino-nucleon scatterings as well as the tau-lepton energy loss. We specifically considered the Earth-skimming situation and provided detailed results for the energy dependencies of emerging tau-lepton fluxes resulting from a few anticipated astrophysical neutrino fluxes. The effect of matter density on the tau-lepton flux is also studied. Such an effect is found to be related to the spectrum index of 
incident neutrino flux. Our treatment thus provides a basis for a more complete and realistic assessment of high-energy-neutrino flux measurements in the under-construction/planning large neutrino telescopes.

\section{Acknowledgements}

We thank N. La Barbera for communicating to us his Monte-Carlo-based results. HA thanks Physics Division of NCTS for support. MAH is supported by Taiwan's Ministry of Education under Research Excellence Project on Cosmology and Particle Astrophysics: Sub-project II with the grant number 92-N-FA01-1-4-2. FFL, GLL, JJT and TWY are supported by National Science Council of Taiwan under the grant numbers NSC91-2112M009-019 and NSC91-2112-M-001-024.

\section{References}

[1] S. W. Barwick, Astroparticle physics with high energy neutrinos, lectures presented at 28th SLAC Summer Institute on Particle Physics: Neutrinos from the Lab, the Sun, and the Cosmos (SSI 2000), Stanford, California, USA, 14-25 Aug 2000; H. Athar, arXiv:hep-ph/0209130. For a recent review article, see H. Athar, arXiv:hep-ph/0212387.

[2] G. Domokos and S. Kovesi-Domokos, arXiv:hep-ph/9801362; arXiv:hep-ph/9805221. See also, D. Fargion, Astrophys. J. 570, 909 (2002) [arXiv:astro-ph/0002453].

[3] X. Bertou, P. Billoir, O. Deligny, C. Lachaud and A. Letessier-Selvon, Astropart. Phys. 17, 183 (2002) [arXiv:astro-ph/0104452].

[4] J. L. Feng, P. Fisher, F. Wilczek and T. M. Yu, Phys. Rev. Lett. 88, 161102 (2002) [arXiv:hep-ph/0105067].

[5] S. Bottai and S. Giurgola, Astropart. Phys. 18, 539 (2003) [arXiv:astro-ph/0205325].

[6] I. Kravchenko et al. [RICE Collaboration], arXiv:astro-ph/0306408. See also, M. Chiba et al., AIP Conf. Proc. 579, 204 (2001).

[7] http://www.ps.uci.edu/ anita/.

[8] L. V. Volkova, G. T. Zatsepin and L. A. Kuzmichev, Sov. J. Nucl. Phys. 29, 645 (1979) [Yad. Fiz. 29, 1252 (1979)].

[9] S. I. Dutta, M. H. Reno, I. Sarcevic and D. Seckel, Phys. Rev. D 63, 094020 (2001) [arXiv:hep-ph/0012350].

[10] B. Rossi, High Energy Particles (Prentice Hall, Englewood Cliffs, NJ, USA, 1952).

[11] A. A. Petrukhin and V. V. Shestakov, Can. J. Phys. 46, S377 (1968).

[12] R. P. Kokoulin and A. A. Petrukhin in Proceedings of the XII International Conference on Cosmic Rays (Hobart, Tasmania, Australia, 1971), Vol. 6.

[13] L. B. Bezrukov and E. V. Bugaev, Sov. J. Nucl. Phys. 33, 635 (1981) [Yad. Fiz. 33, 1195 (1981)]. For a recent discussion, see E. V. Bugaev and Y. V. Shlepin, Phys. Rev. D 67, 034027 (2003) [arXiv:hep-ph/0203096].

[14] V. A. Naumov and L. Perrone, Astropart. Phys. 10, 239 (1999) [arXiv:hep-ph/9804301]. 
[15] P. Lipari and T. Stanev, Phys. Rev. D 44, 3543 (1991).

[16] A. Neronov, D. Semikoz, F. Aharonian and O. Kalashev, Phys. Rev. Lett. 89, 051101 (2002) [arXiv:astro-ph/0201410]; O. E. Kalashev, V. A. Kuzmin, D. V. Semikoz and G. Sigl, Phys. Rev. D 66,063004 (2002) [arXiv:hep-ph/0205050].

[17] E. Waxman and J. N. Bahcall, Phys. Rev. Lett. 78, 2292 (1997) [arXiv:astro-ph/9701231]; ibid., Phys. Rev. D 59, 023002 (1999) [arXiv:hep-ph/9807282].

[18] V. S. Berezinsky and G. T. Zatsepin, Phys. Lett. 28B, 423 (1969). For a recent discussion, see R. Engel, D. Seckel and T. Stanev, Phys. Rev. D 64, 093010 (2001) [arXiv:astro-ph/0101216].

[19] H. Athar, Nucl. Phys. Proc. Suppl. 122, 305 (2003) [arXiv:hep-ph/0210244]; H. Athar, K. Cheung, G. -L. Lin and J. -J. Tseng, Astropart. Phys. 18, 581 (2003) [arXiv:hep-ph/0112222].

[20] J. Ahrens et al. [AMANDA Collaboration], Phys. Rev. Lett. 90, 251101 (2003) [arXiv:astro-ph/0303218].

[21] N. La Barbera, private communications.

[22] G. W. Hou and M. A. Huang, Expected performance of a neutrino telescope for seeing $A G N / G C$ behind a mountain, talk given in 1st NCTS Workshop on Astroparticle Physics, Taiwan, 6-9 Dec 2001, published in its proceedings, pp. 105-116, Eds. H. Athar, G. -L. Lin and K. -W. Ng, World Scientific, Singapore (2002) [arXiv:astro-ph/0204145]. See also http://hep1.phys.ntu.edu.tw/nutel/.

[23] M. A. Huang, G. -L. Lin and J. -J. Tseng, work in progress. 